\title{
The impact of COVID-19-related distress on levels of depression, anxiety and quality of life in psychogeriatric patients
}

\author{
Carolin Miklitz ${ }^{1,2} \cdot$ Christine Westerteicher ${ }^{1,2} \cdot$ Sina Lippold $^{1,2} \cdot$ Lena Ochs $^{1,2} \cdot$ Anja Schneider $^{1,2} \cdot$ Klaus Fliessbach $^{1,2}$
}

Received: 10 May 2021 / Accepted: 22 September 2021 / Published online: 13 October 2021

(c) The Author(s), under exclusive licence to Springer-Verlag GmbH Germany 2021

\begin{abstract}
Within the elderly population, psychogeriatric patients may be particularly susceptible to negative mental health effects of the coronavirus crisis. Detailed information about the psychosocial well-being of psychogeriatric patients during the pandemic is still sparse. Here we examined which aspects of subjective experience of the COVID-19 pandemic especially affect levels of depression, anxiety and quality of life in psychogeriatric patients with and without cognitive impairment. A cross-sectional paper survey was conducted during the first German lockdown among patients with a diagnosed psychiatric disorder $(\geq 60$ years) or a diagnosed neurodegenerative disease (regardless of their age) from the department for neurodegenerative diseases and geriatric psychiatry at the University of Bonn. The WHO-5-, GAD-7- and WHOQOL-old score were used to determine levels of depression, anxiety and quality of life. The second part obtained information about the subjective experience of the COVID-19 pandemic. Statistical analysis included among others principal component analysis and multiple linear regression analysis. COVID-19-related, immediate distress was a strong predictor of elevated symptoms of depression, anxiety and a reduced quality of life. COVID-19-related concerns regarding health and financial security, however, were not significantly associated with negative mental health outcomes. The overall prevalence of symptoms of depression $(50.8 \%[95 \%$ CI 43.8-57.6\%]) and anxiety (32.7\% [95\% CI 26.4-39.2\%]) among psychogeriatric patients was high. Our findings indicate that psychogeriatric patients are not significantly affected by COVID-19-related concerns but are primarily suffering from emotional consequences resulting from changed living conditions due to the pandemic.
\end{abstract}

Keywords COVID-19 $\cdot$ Psychogeriatric patients · Depression · Anxiety · Quality of life

\section{Introduction}

The COVID-19 pandemic has led governments around the world to implement unprecedented public health interventions to mitigate the spread of the coronavirus SARS-CoV-2. One early focus was put on isolating populations at increased risk of a poor outcome in case of infection, especially the elderly.

In Germany, the first nationwide contact restrictions were implemented on March 23, 2020 and lasted until May

Carolin Miklitz and Christine Westerteicher contributed equally.

Carolin Miklitz

Carolin.Miklitz@ukbonn.de

1 Department of Neurodegenerative Diseases and Geriatric Psychiatry, University of Bonn, Bonn, Germany

2 German Center for Neurodegenerative Diseases (DZNE), Bonn, Germany
6, 2020. People were urged to reduce social contacts to a minimum. Staying in the public was only allowed alone or with one other person. Many clinics closed their outpatient departments, visitors in clinics, nursing homes and longterm care facilities were not allowed. On December 16, 2020, a second partial lockdown was imposed. Although more and more people were becoming vaccinated against SARS-CoV-2, in spring 2021 Germany was facing a third wave of COVID-19 infections. In view of a rising number of COVID-19 infections strict contact restrictions were again implemented which could be eased in summer 2021.

It was widely predicted that there would be a marked rise in mental health symptoms during the COVID-19 pandemic [1]. According to the diathesis-stress model, it was expected that those with pre-existing mental health conditions would be most impacted by the pandemic $[2,3]$. Contrary to these predictions, a new systematic review and meta-analysis examining longitudinal cohort studies with data before and during the pandemic found no evidence of 
a worsening of mental health symptoms among those with pre-existing mental health conditions during the coronavirus crisis [4].

Already early in the pandemic concerns were expressed about possible negative health consequences of isolating the elderly since prior studies had identified social disconnection as an important risk factor for depression and anxiety among older adults $[5,6]$.

However, approximately 1 year into the COVID-19 pandemic, early data have suggested that older adults may be more resilient to mental health effects resulting from the coronavirus crisis and have better mental health outcomes than expected.

Studies from high-income countries have noted that older adults reported significantly lower percentages of anxiety, depression and post-traumatic stress disorder than younger adults $[7,8]$. These findings are similar to a representative survey from Germany among adults aged 65 years and older. In this survey an unaltered mental well-being during the COVID-19 lockdown was noted [9].

Within the elderly population, however, psychogeriatric patients may be particularly susceptible to negative mental health effects of measures taken to mitigate the spread of the virus. Psychogeriatric patients may have difficulties to adapt to the severe disruptions of daily routines resulting from the pandemic.

Many resources upon which psychogeriatric patients depend have become unavailable: programs at day care centers, therapeutic measures, home support for personal care and home visits from relatives and friends have been largely suspended. Most social activities have become unfeasible. The loss of daily routines and support may lead to an increase in loneliness and to a rise of psychiatric symptoms.

While all these changes may affect psychogeriatric patients in general, additional concerns regarding people with Alzheimer's disease and other dementias have been raised. People with dementia have difficulties in understanding public health information and safeguard procedures and are often not able to adapt to new routines and techniques, such as the use of telecommunication [10]. Studies indicate that patients with dementia are not only at increased risk of infection, and once infected more likely to experience severe virus-related outcomes, but are also at high risk of experiencing a worsening of neuropsychiatric symptoms as a consequence of social distancing during the pandemic $[11,12]$.

Prior research regarding specifically the mental health outcome of psychogeriatric patients has provided contradictory results. One study of older adults with pre-existing depression did not report an increase in symptoms of depression and anxiety within this group [13] whereas Li et al. noted that among clinically stable psychogeriatric patients prevalence of depression and anxiety during the pandemic were higher than in other age groups [14].
However, detailed information about the psychosocial well-being of psychogeriatric patients during the coronavirus crisis is sparse.

Against this background, we aimed to investigate the prevalence of mental health symptoms and the quality of life during the pandemic in different subgroups of a psychogeriatric population with and without cognitive impairment and examined associated risk factors as well as protective factors. We further addressed the question which aspects of subjective experience of the COVID-19 pandemic particularly affect levels of depression, anxiety and quality of life during the pandemic.

\section{Methods}

\section{Study design}

Data were obtained between April 17, and June 30, 2020. 922 patients and their relatives from the department for neurodegenerative diseases and psychogeriatrics at the University of Bonn were contacted by mail and received detailed information about the study. We contacted all psychogeriatric patients who were treated in the outpatient clinic within the last year (1.04.2019-31.03.2020) as well as all psychogeriatric patients who were hospitalized in our department within the past 3 months (01.01.2020-31.03.2020). Participants received a paper survey and were asked to send the completed questionnaires back by mail.

Medical information was obtained from the medical records of the University Hospital of Bonn. Results from Mini Mental State Exams were used as additional information if administrated within the last 9 months.

\section{Eligibility criteria}

\section{Patients}

Patients 60 years and older with a diagnosed psychiatric disorder and patients with a diagnosed neurodegenerative disease regardless of their age were included.

\section{Relatives}

Inclusion criteria were an age older than 18 years and the ability to complete the questionnaire in German. There were no further inclusion or exclusion criteria.

\section{Measurements}

This paper survey consisted of three parts. The first part recorded demographic information of the participants including age, gender, number of children and grandchildren, 
marital status, level of education, household size and type of accommodation. The amount of support needed was rated on a 4-point Likert scale by the relatives. The second part comprised COVID-19-related questions evaluating information habits, changes in daily life and questions regarding the subjective experience of the COVID-19 pandemic. The questions were rated on a 4-point Likert scale.

The third part consisted of three well-established, standardized scales: (1) the World Health Organization-Five Well-Being Index (WHO-5), a five-item scale assessing psychological well-being which is widely accepted as a specific and sensitive screening tool for depression with ranges from 0 to 100 with 100 representing the best possible well-being status [15]. (2) The seven-item Generalized Anxiety Disorder-7-scale (GAD-7) to assess symptoms of general anxiety (range 0-21 with higher values indicating higher symptoms of anxiety). (3) The WHOQOL-old, a test designed to assess the subjective quality of life of older adults ( $>60$ years) including the six facets sensory abilities, autonomy, satisfaction about activities in the past, present and future, social participation, fears concerning death and dying, and intimacy with a range from 0 to 100 and higher scores representing better quality of life $[16,17]$.

In this study cutoff scores of $<50$ for the World Health Organization-Five Well-Being Index (WHO-5) and $\geq 10$ for the Generalized Anxiety Disorder-7 scale (GAD-7) were applied to define the presence of depressive symptoms [15] and moderate to severe levels of anxiety [18].

\section{Clinical data}

Furthermore, we differentiated between main and secondary psychiatric diagnoses. Dementia was always considered as main diagnosis when co-occurring with other psychiatric disorders. Four groups of main diagnoses were formed: Dementia (including dementia due to Alzheimer's disease, frontotemporal lobe degeneration, Lewy body dementia, normal pressure hydrocephalus (NPH), vascular pathology or mixed etiology), mild cognitive impairment/ subjective cognitive decline (MCI/SCD), mood disorder (depressive, bipolar or schizoaffective) and others (including among others anxiety disorders, addiction, personality disorders, grouped together due to low numbers in single subcategories). For further analysis, we performed two subgroup analyses: (1) we analyzed data from all patients without a pre-described mood disorder regardless of their main diagnosis. (2) We distinguished between patients with cognitive impairment (MCI/SCD or dementia) or other psychiatric disorders (mood disorders and others). Patients with dementia were assigned to three groups according to their last Mini-Mental Status Examination score (MMSE): mild dementia (MMSE $\geq 20$ ), moderate dementia (MMSE <20) and dementia with unknown severity (no recent MMSE available).

\section{Statistical analysis}

The Kolmogorov-Smirnov test was used to assess whether data were normally distributed. Qualitative variables were expressed as percentages and the $95 \%$ confidence intervals [CI] were calculated by bootstrapping with 1000 samples.

Principal component analysis including data from both patients and relatives was used to determine the factor structure of the questionnaire of subjective experience of the COVID-19 pandemic. The analysis was run on the six items of the questionnaire with orthogonal rotation (varimax). The Kaiser-Mayer-Olkin measure was used to verify the sampling adequacy for the analysis. An initial analysis was run to obtain eigenvalues of each factor in the data. Only factors with eigenvalues $>1$ were considered.

The prevalence of symptoms of depression and anxiety was calculated using the aforementioned cut-off scores and was compared in different populations by $\chi^{2}$ tests. $T$ test and one-way ANOVA with Tukey post hoc test were performed to compare normally distributed scores between groups. Otherwise the non-parametric Mann-Whitney or Kruskal-Wallis test with Dunn-Bonferroni post hoc test was applied.

The predictive values of COVID-19-related immediate emotional well-being and concerns [factors retrieved from the questionnaire of subjective experience of the COVID19 pandemic, in the following called Corona-Factor 1 (CF1) and Corona-Factor 2 (CF2)] and various other independent variables (demographic data, diagnosis, number of chronic diseases, intake of antidepressant medication, support in daily life, access to digital media) on depression (WHO-5 score), anxiety (GAD-7 score) and quality of life (WHOQOL-old score) during the pandemic were tested by multiple linear regression analyses. Because of a high correlation between marital status and household size, only the variable household size was included.

In a second model of multiple linear regression analysis predictive factors of elevated levels of self-reported COVID-19-related immediate emotional distress (CF1) were examined. Independent variables included demographic data, diagnosis, number of chronic diseases, intake of antidepressant medication, support in daily life, access to digital media as well as aspects specific to the pandemic: change in amount of support, change in daily routines and information habits.

The level of significance was set to $p<0.05$. All statistical analyses were performed using SPSS statistical software version 25 (IBM Corp). 


\section{Results}

\section{Sample characteristics}

A total of 219 patients completed the survey (response rate $23.8 \%$ ). Main diagnoses were: dementia (95 [43\%]), mild cognitive impairment (MCI) or subjective cognitive decline (SCD) (28 [13\%]), mood disorder (81 [37\%]) and other psychiatric disorders (15 [7\%]). The majority of patients with dementia had a diagnosis of Alzheimer's disease (66 [69.5\%]), followed by frontotemporal dementia $(10[10.5 \%])$, mixed dementia (9 [9.5\%]), Lewy body dementia (4 [4.2\%]) and others (6 [6.3\%]). Most patients with a mood disorder had a diagnosis of a depression (69 [85\%]), followed by bipolar disorder (10 [12\%]) and schizoaffective disorder (2 [2\%]). Within the group of patients with MCI/SCD 15 [54\%] patients had a diagnosis of mild cognitive impairment, 13 [46\%] patients had the diagnosis of subjective cognitive decline. 105 patients were male [48\%] and patients' mean $[S D]$ age was 73.04 [0.58] years. Of 158 patients a corresponding relative completed the COVID-19-related mental health consequences and concerns questionnaire, too. Relatives were on average [SD] 67.58 [0.94] years old, $43.7 \%$ were male.

A recent Mini-Mental Status Examination score (MMSE) was available for 66 [69.5\%] patients with dementia. According to the MMSE score, 45 [68.2\%] patients were classified as "mild dementia" and 21 [31.8\%] as "moderate". 16 [16.8\%] patients with dementia had a pre-described mood disorder. Detailed characteristics of all participants are presented in Table 1.

\section{High prevalence of depression and anxiety}

The prevalence of depressive symptoms among all psychogeriatric patients was 50.8\% (95\% CI 43.8-57.6\%), the prevalence of moderate to severe levels of anxiety was $32.7 \%$ (95\% CI 26.4-39.2\%). The prevalence of elevated levels of anxiety was significantly higher in patients with mood disorders $(41.6 \%, 95 \%$ CI $29.7-52.5 \%)$ compared to patients with dementia $(28.2 \%, 95 \%$ CI $17.5-38.6 \%$, $p=0.041)$ or $\mathrm{MCI} / \mathrm{SCD}(17.9 \%, 95 \%$ CI $4.3-34.5 \%$, $p=0.026$ ). The prevalence of depressive symptoms was not significantly different between the groups (mood disorder $53.8 \%, 95 \%$ CI $42.5-63.7 \%$; dementia $44.4 \%, 95 \%$ CI $34.6-55.7 \%$; MCI/SCD $53.6 \%, 95 \%$ CI $35.7-71.4 \%$; $\left.\chi^{2}(2)=1.73, p=0.503\right)$.

The overall median [IQR] GAD-7 score among all psychogeriatric patients was 6.0 [8.0], the median WHO-5 score was 48.0 [48]. For patients with dementia, mood disorder and $\mathrm{MCI} / \mathrm{SCD}$ the median GAD-7 score was 6.0
[8.5], 7.0 [9.25] and 4.5 [6.75], respectively. The median WHO-5 score was 56.0 [44.0] for patients with dementia, 44.0 [52.0] for patients with mood disorder and 48.0 [37.0] for patients with MCI/SCD. The European Quality of Life Survey 2016 reported a mean WHO-5 score of 65.0 among the general elderly German population aged 65 years and older [19]. A normative examination of the GAD-7 questionnaire among the general German population published in 2017 reported a mean GAD-7 score of 3.57 (SD 3.38) without a substantial age trend [20].

Both median GAD-7 score $\left[\chi^{2}(3)=4.72, p=0.194\right]$ and WHO-5 score $\left[\chi^{2}(3)=2.98, p=0.395\right]$ were not significantly different between the groups (mood disorder, dementia, $\mathrm{MCI} / \mathrm{SCD}$ and other psychiatric disorders).

Regarding all patients with cognitive impairment and other psychogeriatric disorders and excluding all those with a pre-described mood disorder $(n=106)$ the prevalence of depressive symptoms remained high $(43.6 \%, 95 \% \mathrm{CI}$ $33.7-53.1 \%$ ). The prevalence of moderate to severe symptoms of anxiety was $23.8 \%$ [ $95 \%$ CI $15.7-32.7 \%$ ].

Among patients without pre-described mood disorder the median $[I Q R]$ GAD-7 score was 5.0 [7.0], the median WHO-5 score was 56.0 [44.0].

\section{Poorer quality of life in patients with mood disorder}

The mean [SD] WHOQOL-old-score measuring quality of life during the COVID-19 pandemic was 63.91 [14.41] without significant differences with respect to gender and/ or main diagnosis. Regarding all patients with mood disorders (as main or secondary diagnosis) patients with a pre-described mood disorder had significant lower mean WHOQOL-old-scores (60.48 [15.27]) compared to patients without mood disorders (66.98 [12.93], $p=0.001)$. In a representative sample of the German elderly population from 2012 the mean WHOQOL-old score was 68.42 [SE $=0.52$ ]. In elderly people diagnosed with depression, who were also part of the German WHOQOL standardization process the mean WHOQOL-old score was 63.51 [SE $=1.19$ ] [21].

\section{Subjective experience of the COVID-19 pandemic: immediate emotional well-being, health worries and financial concerns}

46.9\% [95\% CI 40.3-54.8\%] of patients reported an increase in loneliness and 38.9\% [95\% CI 32.5-45.8\%] felt an increase in sadness due to the coronavirus crisis. 48.8\% [95\% CI 42.0-55.7\%] agreed that the pandemic caused anxiety. Evaluating specific concerns regarding the pandemic the majority of patients were concerned to develop severe complications from an infection with COVID-19 (53.6\%, 95\% CI 46.9-60.7\%) and 54.5\% [95\% CI 47.9-61.1\%] were afraid about a probable severe course 
Table 1 Demographic characteristics

\begin{tabular}{|c|c|c|c|c|c|c|}
\hline & \multicolumn{4}{|c|}{ Main diagnosis } & \multirow[t]{2}{*}{$p$} & \multirow{2}{*}{$\begin{array}{l}\text { All patients } \\
N=219\end{array}$} \\
\hline & $\begin{array}{l}\text { Dementia } \\
n=95\end{array}$ & $\begin{array}{l}\text { SCD/MCI } \\
n=28\end{array}$ & $\begin{array}{l}\text { Mood disorder } \\
n=81\end{array}$ & $\begin{array}{l}\text { Others } \\
n=15\end{array}$ & & \\
\hline \multirow[t]{2}{*}{ Age (years) } & 72.83 & 73.64 & 72.67 & 76.8 & 0.768 & 73.04 \\
\hline & {$[9.16]$} & {$[9.29]$} & {$[7.68]$} & [7.13] & & {$[0.58]$} \\
\hline Gender (ref. male) & $50.0 \%$ & $46.4 \%$ & $49.4 \%$ & $33.3 \%$ & 0.675 & $48.0 \%$ \\
\hline Single-person household & $11.0 \%$ & $35.7 \%$ & $35.1 \%$ & $35.7 \%$ & 0.001 & $25.4 \%$ \\
\hline Marital status married or firm partnership & $90.4 \%$ & $78.6 \%$ & $65.4 \%$ & $53.3 \%$ & $<0.001$ & $76.0 \%$ \\
\hline Appartment size $\geq 3-4$ room appartment & $90.4 \%$ & $82.1 \%$ & $78.5 \%$ & $66.7 \%$ & 0.051 & $83.1 \%$ \\
\hline Level of education $\geq$ enrolment standard & $32.3 \%$ & $28.6 \%$ & $32.1 \%$ & $46.7 \%$ & 0.667 & $32.3 \%$ \\
\hline Antidepressant medication & $34.7 \%$ & $14.3 \%$ & $79.0 \%$ & $60.0 \%$ & $<0.001$ & $49.5 \%$ \\
\hline \multicolumn{7}{|l|}{ Chronic somatic diseases } \\
\hline More than 3 & $16.8 \%$ & $42.9 \%$ & $30.9 \%$ & $6.7 \%$ & 0.006 & $24.3 \%$ \\
\hline Unknown & $10.5 \%$ & $0.0 \%$ & $1.2 \%$ & $20.0 \%$ & 0.005 & $7.7 \%$ \\
\hline \multicolumn{7}{|l|}{ Support in every day life (ref. no or little) } \\
\hline Sometimes & $25.3 \%$ & $46.4 \%$ & $25.9 \%$ & $0.0 \%$ & 0.011 & $26.1 \%$ \\
\hline Often & $32.6 \%$ & $14.3 \%$ & $18.5 \%$ & $6.7 \%$ & 0.026 & $23.0 \%$ \\
\hline Always & $23.2 \%$ & $0.0 \%$ & $6.2 \%$ & $6.7 \%$ & 0.001 & $12.6 \%$ \\
\hline Unknown & $10.5 \%$ & $28.6 \%$ & $32.1 \%$ & $66.7 \%$ & $<0.001$ & $25.7 \%$ \\
\hline Support by family & $47.3 \%$ & $42.9 \%$ & $56.8 \%$ & $66.7 \%$ & 0.286 & $51.4 \%$ \\
\hline Support by friends & $10.8 \%$ & $10.7 \%$ & $24.7 \%$ & $40.0 \%$ & 0.008 & $18.2 \%$ \\
\hline Professional medical support & $12.9 \%$ & $17.2 \%$ & $31.0 \%$ & $20.0 \%$ & 0.698 & $13.2 \%$ \\
\hline \multicolumn{7}{|l|}{ Change in amount of support } \\
\hline Increase & $22.6 \%$ & $23.1 \%$ & $23.8 \%$ & $28.6 \%$ & 0.969 & $23.1 \%$ \\
\hline Decrease & $12.9 \%$ & $3.8 \%$ & $12.5 \%$ & $21.4 \%$ & 0.414 & $12.0 \%$ \\
\hline Access to digital media & $79.8 \%$ & $89.3 \%$ & $90.0 \%$ & $86.7 \%$ & 0.265 & $85.1 \%$ \\
\hline Information about COVID-19 via newspaper & $65.2 \%$ & $57.1 \%$ & $66.3 \%$ & $60.0 \%$ & 0.823 & $64.7 \%$ \\
\hline Information about COVID-19 via Internet & $20.7 \%$ & $39.3 \%$ & $26.3 \%$ & $53.3 \%$ & 0.027 & $27.5 \%$ \\
\hline Change of daily routines due to COVID-19 & $63.0 \%$ & $75.0 \%$ & $74.1 \%$ & $73.3 \%$ & 0.375 & $69.9 \%$ \\
\hline \multicolumn{7}{|l|}{ COVID-19-contact } \\
\hline Positive COVID-19 Test & $0.0 \%$ & $0.0 \%$ & $1.3 \%$ & $0.0 \%$ & 0.649 & $0.5 \%$ \\
\hline Quarantine & $1.1 \%$ & $0.0 \%$ & $3.8 \%$ & $13.3 \%$ & 0.047 & $2.9 \%$ \\
\hline Close persons with COVID-19 infection & $1.1 \%$ & $3.8 \%$ & $8.8 \%$ & $0.0 \%$ & 0.078 & $4.3 \%$ \\
\hline Self-preception as COVID-19 risk group & $69.9 \%$ & $88.9 \%$ & $86.1 \%$ & $80.0 \%$ & 0.035 & $79.3 \%$ \\
\hline Completion of questionnaire during lockdown & $56.8 \%$ & $64.3 \%$ & $67.9 \%$ & $73.3 \%$ & 0.701 & $63.10 \%$ \\
\hline
\end{tabular}

$p$-values $<0.05$ were regarded as significant and are shown in bold

of a coronavirus infection of close persons. Only $15.6 \%$ [95\% CI 10.9-20.8\%] were concerned about potential financial losses due to the coronavirus crisis. Between the groups (dementia, MCI/SCD, mood disorder and other psychiatric disorder) there were no significant differences in the prevalence of the aforementioned COVID-19-related emotional consequences and concerns.

To further determine the factor structure of the questionnaire of subjective experience of the COVID-19 pandemic a principal component analysis was run with orthogonal rotation (varimax). The Kaiser-Meyer-Olkin measure verified the sampling adequacy for the analysis $(\mathrm{KMO}=0.65)$.
Examination of Kaiser's criteria and the screen-plot yielded empirical justification for retaining two factors with eigenvalues exceeding 1 , which accounted for 60.31 of the total variance. The first factor comprises the anxiety resulting from the pandemic, an increase in sadness during COVID-19 and an increase in loneliness as a result of the precautions taken to contain the coronavirus crisis. We specify these parameters as Corona Factor 1 (CF1) which taken together describes the immediate COVID-19-related emotional distress. Concerns about a severe personal clinical course of the disease, about a probable severe infection of close persons and financial concerns due to COVID-19 constitute the second factor (Corona 
Table 2 Results from the principal component analysis of the questionnaire of subjective experience of the COVID-19 pandemic

Item

Rotated factor loadings

Emotional Concerns

distress

(CF1)

(CF2)

Do you feel that the coronavirus crisis has had a negative impact on your mood? Are you sad more often than before?

0.89

Do you feel lonelier as a result of the precautions taken to contain the corona virus crisis?

0.83

Does the corona virus crisis cause you anxiety?

0.73

Are you concerned that you would develop severe complications from a coronavirus infection (Covid-19)?

0.8

Are you concerned that people close to you could become seriously ill with a coronavirus infection (Covid-19)?

Are you concerned about potential financial losses due to the coronavirus crisis?

Factor 2, CF2). This CF2 thus describes COVID-19-related concrete concerns and worries. In the following CF1 is referred to as "COVID-19-related immediate emotional distress", CF2 as "COVID-19-related concerns". Table 2 shows the factor loadings after rotation.

Analyzing the resulting values of CF1 and CF2 in respect to main diagnosis, gender and age ( $>60$ years vs. others) did not reveal any significant differences between the groups (CF1: $p=0.520,0.570$ and $0.981 ; \mathrm{CF} 2: p=0.301,0.109$ and 0.804 , respectively). Patients with a pre-described mood disorder (as main or secondary diagnosis) reported significantly higher levels $(M=0.05, \mathrm{SD}=0.91)$ of COVID-19-related concerns (CF2) than patients without pre-described mood disorder $(M=-0.23, \mathrm{SD}=0.99, t(209)=-2.12, p=0.035)$. No significant differences in the self-reported levels of COVID-19-related immediate emotional well-being (CF1) between patients with $(M=0.13, \mathrm{SD}=1.02)$ and without pre-described mood disorder $(M=-0.11, \mathrm{SD}=0.92)$ were found $[t(209)=-1.84$, $p=0.067]$.

\section{No differences in respect to the timepoint of completion of the questionnaire}

Considering the timepoint of completion of the questionnaire, there were no significant differences found between the mean WHOQOL-score $[t(202)=-0.40, p=0.970]$ and median WHO-5 $(U=0.012, p=0.994)$ and GAD-7 scores $(U=2.45$, $p=0.288$ ) between patients who completed the questionnaire during and after the first German lockdown (from March 23 until May 6, 2020).
CF1 and CF2 did not significantly differ between patients who completed the questionnaire during or after the lockdown (CF1: $t=-0.45, p=0.967, \mathrm{CF} 2: t=0.455, p=0.657$ ), either.

\section{Self-reported COVID-19-related immediate emotional distress (CF1) rather than COVID-19 concerns (CF2) are associated with negative mental health outcomes}

The multiple linear regression models 1a-c including different independent variables (demographic data, diagnosis, support in daily life, information habits, use of digital media, CF1 and CF2) identified self-reported COVID19-related immediate, emotional distress (CF1) as a strong predictor of elevated depressive symptoms (WHO-5 score, $t=-6.27, p<0.001$ ), higher levels of anxiety (GAD-7, $t=7.23, p<0.001$ ) and of a poorer quality of life (WHOQOL-old, $t=-5.95, p<0.001)$. COVID-19-related concerns (CF2), however, were not significantly associated with the aforementioned scales of depression, anxiety and quality of life ( $p=0.681, p=0.241$ and $p=0.053)$.

Being entirely dependent on support from others was also a predictor of higher levels of depressive symptoms $(t=-2.41, p=0.021)$, anxiety $(t=3.44, p=0.001)$ and of a poorer quality of life $(t=-3.24, p=0.001)$. Access to digital media was identified as a positive predictor of a higher quality of life $(t=3.52, p=0.001)$ and of lower levels of depressive symptoms $(t=2.41, p=0.021)$. Table 3 provides information about all variables included in the multiple linear regression models $1 \mathrm{a}-\mathrm{c}$. 


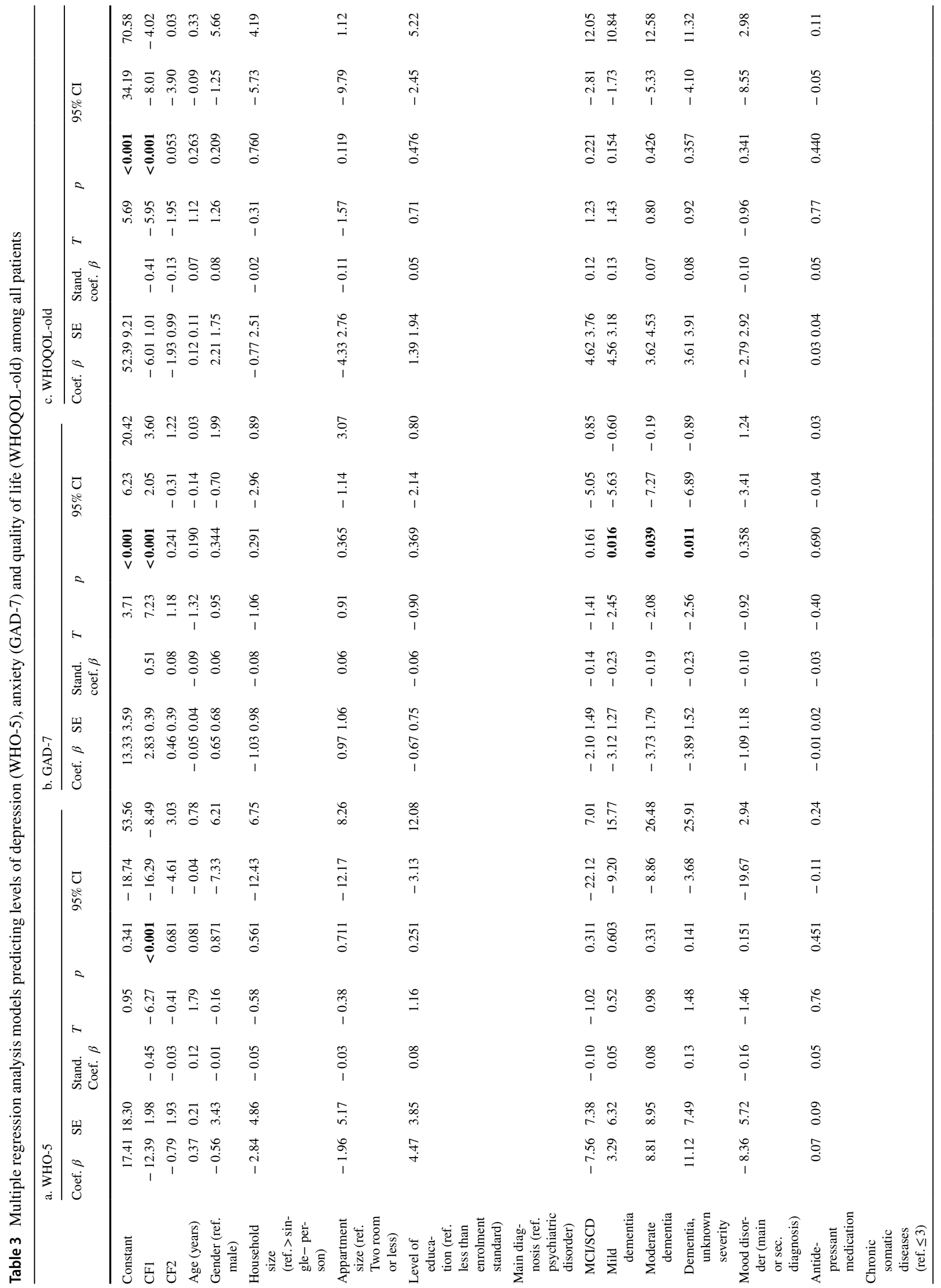




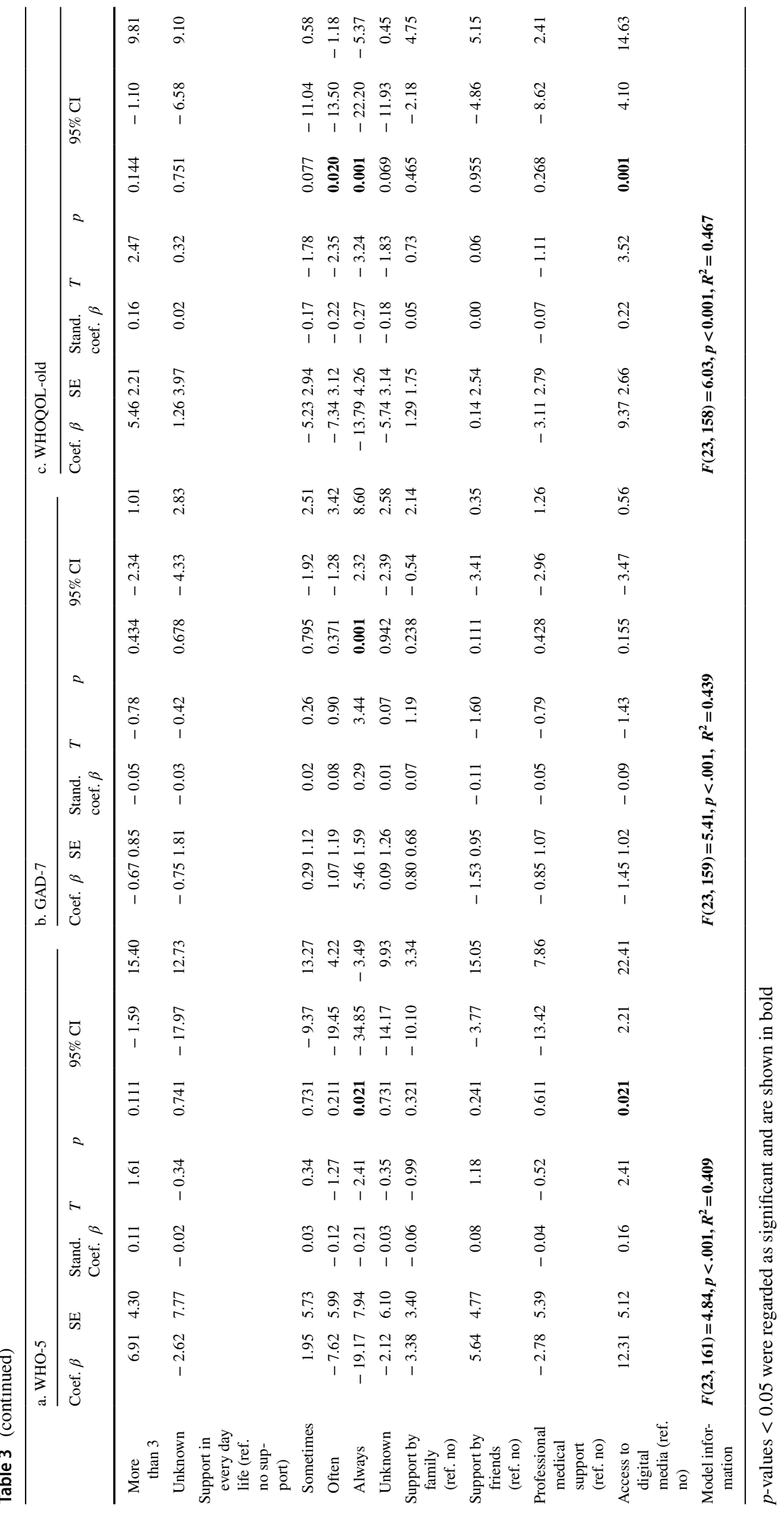




\section{Predictors of elevated self-reported COVID-19-related sadness, loneliness and anxiety: Internet use is a protective factor}

In a second multiple linear regression model we analyzed predictors of self-reported COVID-19-related immediate, emotional distress (CF1 being used as the response variable in this analysis). Patients who reported a change of daily routines due to the COVID-19 pandemic were more likely to experience higher levels of COVID-19-related immediate distress (CF1) $(t=4.62, p<0.001)$. Both an increase $(t=3.68, p<0.001)$ and a decrease $(t=2.77, p=0.006)$ in the amount of daily support was associated with a negative impact on the emotional state. Hereby, support from family members $(t=-2.66$, $p=0.009$ ) was a predictor of reduced self-reported sadness, loneliness and anxiety. Being in need of assistance in most or all activities of daily life also significantly predicted elevated levels of Corona Factor $1(t=2.06, p=0.041)$. Living alone $(t=2.31, p=0.022)$ was a risk factor for elevated COVID19-related immediate distress (CF1) in patients, too. Access to information about the COVID-19 pandemic via internet $(t=-2.29, p=0.023)$ was associated with lower levels of COVID-19-related distress. Detailed information about the variables included in the multiple linear regression model 2 predicting CF1 are shown in Table 4.

\section{Patients with moderate dementia report lower quality of life and higher levels of COVID-19-related emotional distress (CF1)}

Subsequently, we examined the mental health outcomes of patients with cognitive impairment in detail.

After correcting for age, gender, the condition of a pre-described mood disorder and pre-existing chronic

Table 4 Multiple regression analysis model predicting the level of COVID-19-related, immediate emotional distress (CF1) among all patients

\begin{tabular}{|c|c|c|c|c|c|c|}
\hline & COVID-19 related & notional distress & $\mathrm{F} 1)$ & & & \\
\hline & Coef. $\beta$ & stand. coef. $\beta$ & $T$ & $p$ & $95 \% \mathrm{CI}$ & \\
\hline Costant & -0.090 .63 & & -0.14 & 0.889 & -1.33 & 1.15 \\
\hline Age (years) & -0.010 .01 & -0.05 & -0.74 & 0.458 & -0.02 & 0.01 \\
\hline Gender (ref. male) & -0.170 .12 & -0.08 & -1.37 & 0.172 & -0.41 & 0.07 \\
\hline Household size (ref. > single-person) & 0.400 .17 & 0.18 & 2.31 & 0.022 & 0.06 & 0.75 \\
\hline Appartment size (ref. Two room or less) & 0.030 .19 & 0.01 & 0.13 & 0.893 & -0.35 & 0.40 \\
\hline Level of education (ref. less than enrolment standard) & 0.050 .14 & 0.02 & 0.33 & 0.739 & -0.23 & 0.32 \\
\hline Main diagnosis (ref. psychiatric disorder) & & & & & & \\
\hline $\mathrm{MCI} / \mathrm{SCD}$ & -0.340 .19 & -0.12 & -1.81 & 0.073 & -0.71 & 0.03 \\
\hline Mild dementia & -0.080 .16 & -0.03 & -0.50 & 0.620 & -0.40 & 0.24 \\
\hline Moderate dementia & -0.170 .26 & -0.05 & -0.66 & 0.512 & -0.68 & 0.34 \\
\hline Dementia, unknown severity & -0.030 .22 & -0.01 & -0.12 & 0.903 & -0.46 & 0.41 \\
\hline Antidepressant medication (ref. no) & 0.230 .16 & 0.10 & 1.45 & 0.148 & -0.08 & 0.54 \\
\hline Chronic somatic diseases (ref. $\leq 3$ ) & & & & & & \\
\hline More than 3 & 0.340 .26 & 0.08 & 1.30 & 0.197 & -0.18 & 0.86 \\
\hline Unknown & 0.000 .00 & 0.10 & 1.63 & 0.105 & 0.00 & 0.01 \\
\hline Support in every day life (ref. no or little) & & & & & & \\
\hline$\geq$ Most of the time & 0.330 .16 & 0.16 & 2.06 & 0.041 & 0.01 & 0.64 \\
\hline Unknown & -0.080 .18 & -0.03 & -0.43 & 0.669 & -0.43 & 0.27 \\
\hline Support by family (ref. no) & -0.330 .12 & -0.17 & -2.66 & 0.009 & -0.57 & -0.08 \\
\hline Support by friends (ref. no) & 0.170 .17 & 0.07 & 0.97 & 0.331 & -0.17 & 0.51 \\
\hline Professional medical support (ref. no) & 0.200 .19 & 0.07 & 1.06 & 0.291 & -0.17 & 0.58 \\
\hline Change in amount of support (ref. no) & & & & & & \\
\hline Increase & 0.550 .15 & 0.24 & 3.68 & $<0.001$ & 0.25 & 0.84 \\
\hline Decrease & 0.550 .20 & 0.18 & 2.77 & 0.006 & 0.16 & 0.94 \\
\hline Access to digital media (ref. no) & -0.040 .18 & -0.01 & -0.22 & 0.830 & -0.40 & 0.32 \\
\hline Information about COVID-19 via newspaper (ref. no) & -0.100 .14 & -0.05 & -0.72 & 0.475 & -0.39 & 0.18 \\
\hline Information about COVID-19 via Internet (ref. no) & -0.330 .15 & -0.15 & -2.29 & 0.023 & -0.62 & -0.05 \\
\hline Change of daily routines due to COVID-19 (ref. no) & 0.640 .14 & 0.30 & 4.62 & $<0.001$ & 0.37 & 0.91 \\
\hline Model information & $F(23,167)=4.84$, & $<.001, R^{2}=0.4$ & & & & \\
\hline
\end{tabular}

$p$-values $<0.05$ were regarded as significant and are shown in bold 
Table 5 Multiple regression analysis model predicting the level of COVID-19-related, immediate emotional distress (CF1) among patients with cognitive impairment

\begin{tabular}{|c|c|c|c|c|c|c|c|}
\hline & COVID- & related & onal distress $(\mathrm{CH}$ & & & & \\
\hline & Coef. $\beta$ & SE & Stand. coef. $\beta$ & $T$ & $p$ & $95 \% \mathrm{CI}$ & \\
\hline Constant & & 650.70 & & 0.92 & 0.357 & -0.75 & 2.05 \\
\hline Age (years) & & 010.01 & -0.12 & -1.24 & 0.219 & -0.03 & 0.01 \\
\hline Gender (ref. male) & & 280.17 & -0.15 & -1.62 & 0.107 & -0.63 & 0.06 \\
\hline Mood disorder (main or sec. Diagnosis, ref. no) & & 320.26 & 0.11 & 1.25 & 0.214 & -0.19 & 0.84 \\
\hline Chronic somatic diseases (ref. $\leq 3$ ) & & & & & & & \\
\hline More than 3 & & 220.21 & 0.10 & 1.01 & 0.315 & -0.21 & 0.64 \\
\hline Unknown & & 400.32 & 0.12 & 1.26 & 0.209 & -0.23 & 1.04 \\
\hline Main diagnosis (ref. $<$ mild dementia) & & & & & & & \\
\hline Moderate dementia & & 540.25 & 0.21 & 2.10 & $\mathbf{0 . 0 3 8}$ & 0.03 & 1.04 \\
\hline Dementia, unknown severity & & 370.22 & 0.16 & 1.71 & 0.091 & -0.06 & 0.80 \\
\hline Model information & $F(7,105$ & $2.42, p$ & $5, R^{2}=0.139$ & & & & \\
\hline
\end{tabular}

$p$-values $<0.05$ were regarded as significant and are shown in bold

somatic diseases the diagnoses moderate dementia $(t=-3.06, p=0.003)$ and dementia with unknown MMSE ( $t=-3.05, p=0.003)$ were significantly associated with a reduced quality of life during the pandemic compared to patients with $\mathrm{MCI} / \mathrm{SCD}[F(8,102)=3.82$, $\left.p=0.001, R^{2}=0.231\right]$. In line with this finding patients with moderate dementia $(M=59.93, \mathrm{SD}=11.04)$ reported a significantly lower quality of life than patients with $\mathrm{MCI} / \mathrm{SCD}(M=71.89, \mathrm{SD}=10.95, p=0.028)$ or mild dementia $[M=69.85, \mathrm{SD}=12.65, p=0.015, F(2$, $68)=4.95, p=0.01]$. Patients with moderate dementia were also more likely to experience higher levels of COVID-19-related immediate, emotional distress (CF 1) than patients with MCI/SCD or mild dementia $(t=2.10$, $p=0.034$, Table 5) and reported significantly higher levels of COVID-19-related immediate, emotional distress (CF1, $M=0.30, \mathrm{SD}=1.04)$ than patients with MCI or mild dementia $[M=-0.26, \mathrm{SD}=0.89, t(71)=-2.20$, $p=0.031]$.

\section{COVID-19-related emotional distress (CF1) predicts depression, anxiety and a reduced quality of life among patients with cognitive impairment, too}

As in psychogeriatric patients in general among patients with cognitive impairment self-reported COVID-19-related immediate, emotional distress (CF 1) was significantly associated with both more symptoms of depression $(t=-4.89, p<0.001)$ and anxiety $(t=5.84, p<0.001)$ and with a reduced quality of life $(t=-3.73, p<0.001)$ during the pandemic (Table 6).
In this subgroup, again access to digital media was a significant predictor $(t=3.42, p=0.001)$ of a higher quality of life during the coronavirus crisis.

\section{Discussion}

In this study, we aimed to investigate the impact of the coronavirus crisis on mental health symptoms and the quality of life of psychogeriatric patients. Previous studies among the general elderly population have suggested that older adults may be more resilient to negative mental health outcomes from the coronavirus crisis [22]. In contrast, our data suggest that patients with psychogeriatric disorders are particularly susceptible to the negative mental health effects of the COVID-19 crisis.

The prevalence of symptoms of depression and anxiety in our group of psychogeriatric patients was high compared to the general population. This is not surprising in a group of patients with psychiatric diseases. The strength of this study, however, is that we also examined the relationship between these symptoms and the subjective experience of the COVID-19 pandemic. Here, our data suggest a differentiated finding: it is the concrete emotional impact of the pandemic in form of an increase in loneliness, sadness and a general anxiety from the coronavirus crisis and not so much the fear of illness or financial concerns which is strongly related to symptoms of depression, anxiety and a poorer quality of life.

In comparison to a previous study among the general German population which reported a prevalence of $34.5 \%$ [95\% CI 30.0-39.5\%] of depressive symptoms as indicated 


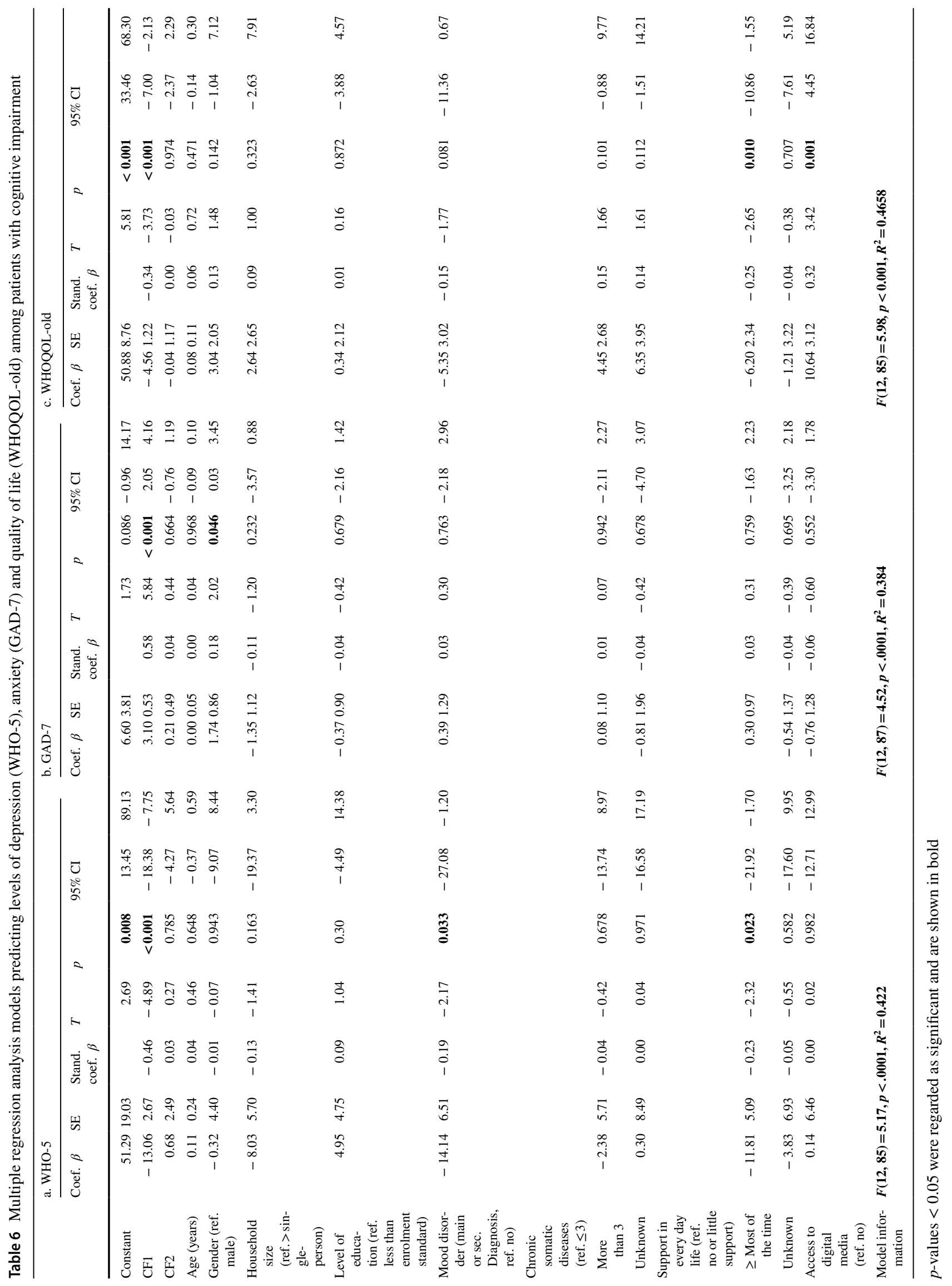


by a WHO-5-score $<50$ in 2018 and of $36.7 \%$ [95\% CI 33.2-40.4] in April 2020 [23] the prevalence of depressive symptoms in our sample was high $(50.8 \%, 95 \% \mathrm{CI}$ 43.8-57.6\%). Also among patients without pre-described mood disorders we found a high prevalence of depressive symptoms $(43.6 \%, 95 \%$ CI $33.7-53.1 \%)$ suggesting that depressive symptoms may have recently emerged. A large cross-sectional online survey among the general German population reported a percentage of $4.4 \%$ (age 65-74) and $5.9 \%$ (age $\geq 75$ ) of participants with a GAD-7 score $\geq 10$ during the initial phase of the pandemic [24]. In comparison, the prevalence of moderate to severe symptoms of anxiety in our sample of psychogeriatric patients was high $(32.7 \%, 95 \%$ CI $26.4-39.2 \%)$.

In our study $46.9 \%$ (95\% CI $40.3-54.8 \%$ ) of patients reported an increase in loneliness as a result of the precautions taken to contain the spread of the coronavirus and $38.9 \%$ (95\% CI 32.5-45.8\%) felt an increase in sadness. Nearly half of the patients $(48.8 \%, 95 \%$ CI $42.0-55.7 \%)$ agreed that the current epidemiological crisis caused them anxiety.

Within an elderly Spanish population without current or previous mental disorders no relationship between loneliness and symptoms of anxiety, depression and stress were found [25]. Van Tilburg et al. reported an increase in loneliness among Dutch older adults while mental health hardly changed. Concerns about the pandemic though, were associated with an increase in mental health problems [26]. In contrast, our study among psychogeriatric patients supports a different conclusion. The self-reported COVID-19-related immediate distress (increase in loneliness, increase in sadness and anxiety from the pandemic, $\mathrm{CF} 1$ ) was a strong predictor of higher levels of depression and anxiety and of a poorer quality of life during the pandemic.

In our study, the majority of patients were concerned that they $(53.6 \%, 95 \%$ CI $46.9-60.7 \%)$ or close persons $(54.5 \%$, 95\% CI 47.9-61.1\%) could develop severe complications from an infection with COVID-19. Only few patients were concerned about potential financial losses attributable to the pandemic which might partly be due to the fact that most patients were no longer in professional life. Also, the high economic background in our sample appears to be high (as e.g. indicated by the apartment size).

While previous studies among the general elderly population noted significant associations between perceived coronavirus threat and mental health outcomes during the pandemic [26, 27] within our sample of psychogeriatric patients COVID-19-related concerns did not significantly predict negative mental health outcomes.

A second aim of this study was the identification of potential risk factors underlying a higher susceptibility to negative mental health outcomes during the pandemic. Patients who reported a change of daily routines due to COVID-19 restrictions were more likely to experience an increase in sadness, loneliness and a general anxiety from the pandemic. Both an increase and a decrease in the amount of daily support were associated with higher levels of COVID-19-related distress indicating that psychogeriatric patients are especially affected by disruptions in their daily routines.

Noteworthily, obtaining information about the pandemic via the internet was a significant predictor of reduced COVID-19-related immediate, emotional distress. Access to digital media was associated with a better quality of life. $27.2 \%$ of patients were already making use of the internet to obtain information about the coronavirus crisis and in total $85.2 \%$ reported having potentially access to digital media. It thus appears that also among psychogeriatric patients there is a remarkable potential to benefit from the advantages of new communication technologies in times of social distancing.

Finally, we addressed the question, whether patients with higher degrees of cognitive impairment were differently affected by the coronavirus crisis. After correcting for potential confounding variables (age, gender, pre-described mood disorder and chronic somatic disease [28]), the diagnosis of moderate dementia was significantly associated with poorer quality of life in comparison to the diagnoses of SCD, MCI and mild dementia.

This is striking, since previous studies did not find a significant association between severity of dementia and quality of life $[29,30]$. Our data therefore suggests that mental health consequences of the ongoing pandemic are disproportionately affecting patients with higher degrees of cognitive impairment. Consistent with this hypothesis, patients with moderate dementia were at higher risk of experiencing elevated levels of COVID-19-related immediate, emotional distress.

The strengths of this study included the well characterized sample with the availability of reliable information from medical records and the paper format of the questionnaires which in contrast to many other recent internet surveys also included patients without internet access.

However, several limitations have to be addressed. First, no recent diagnostic evaluation for depressive or anxiety disorder was conducted, but clinically validated screening instruments were used. Second, there might be a participation bias. Moreover, patients from a specialized university hospital are not representative of community-based psychogeriatric patients which could limit the generalization of the findings, too. Third, the sample size, especially of the group of patients with moderate dementia, was limited. Fourth, our study design and outcome measures were different from previous studies; therefore, the possibility of comparing our results with literature is limited. Fifth, a reverse causation 
between levels of depression, anxiety and quality of life, and COVID-19-related immediate, emotional distress (CF1) cannot be ruled out. Finally, due to the cross-sectional design, the general interpretation of causal relationships between symptoms of depression and anxiety and quality of life with other variables is limited. Further longitudinal research is needed.

In conclusion, our data indicate that psychogeriatric patients are differently affected by the coronavirus crisis than the general elderly population. Our findings suggest that mental health outcomes among psychogeriatric patients are predominantly influenced by emotional reactions to the current pandemic and its implications on every-day life. COVID-19-related specific concerns, however, are not associated with negative mental health consequences. Unlike the general elderly population psychogeriatric patients are particular susceptible to negative mental health outcomes during the pandemic. The prevalence of symptoms of depression and anxiety is high. Among psychogeriatric patients those with higher degrees of cognitive impairment seem to be at the highest risk of experiencing negative mental health consequences from the coronavirus crisis.

One year into the pandemic with more and more elderly persons becoming vaccinated against SARS-CoV-2, these findings are particularly interesting. While a personal vaccination might reduce the perceived coronavirus threat, disruptions in every-day life will probably persist for several more months. By now, we do not expect social distancing rules to be completely abandoned in the near future. Our findings indicate that psychogeriatric patients are not significantly affected by COVID-19-related concerns but are primarily suffering from emotional consequences resulting from non-pharmaceutical preventive measures taken to contain the pandemic. It is, therefore, unlikely that the observed mental health consequences from the pandemic can be resolved by vaccinations alone, as long as containment measures are maintained. However, the newly adopted exemptions from certain COVID-19 restrictions for fully vaccinated people might bring a first relief.

\section{Conclusion}

Self-reported COVID-19-related, immediate and predominantly emotional sequelae have a significant negative effect on depression, anxiety and quality of life in psychogeriatric patients during the pandemic. COVID-19-related concerns regarding health and financial security, however, seem to be of lesser importance. Among psychogeriatric patients those with higher degrees of cognitive impairment seem to be at the highest risk of experiencing negative mental health consequences from the coronavirus crisis.
In times of social distancing, access to digital media is an important positive predictor for a higher quality of life in psychogeriatric patients.

Supplementary Information The online version contains supplementary material available at https://doi.org/10.1007/s00406-021-01340-1.

Author contributions All authors contributed to the study conception and design. Material preparation, data collection and analysis were performed by CM, CW, KF, SL and LO. The draft of the manuscript was written by $\mathrm{CM}, \mathrm{CW}$ and $\mathrm{KF}$ and all authors commented on previous versions of the manuscript. All authors read and approved the final manuscript.

Funding No funding was received for conducting this study.

Availability of data and materials All data and materials as well as software applications used support the published claims and comply with field standards.

Code availability Not applicable.

\section{Declarations}

Conflict of interest The authors declare that they have no conflict of interest.

Ethics approval The questionnaire and methodology for this study was approved by the Human Research Ethics committee of the University of Bonn (Ethics approval number: 139/20).

Consent to participate Informed written consent was received of all participants prior to their inclusion in the study. If patients were incapable of giving their informed consent, written consent of their legally designated representative was obtained.

Consent for publication Patients or their legally designated representatives signed informed consent regarding publishing their data.

\section{References}

1. Pfefferbaum B, North CS (2020) Mental Health and the Covid19 Pandemic. N Engl J Med 6:510-512. https://doi.org/10.1056/ NEJMp2008017

2. Yao H, Chen J-H, Xu YF (2020) Patients with mental health disorders in the COVID-19 epidemic. Lancet Psychiatry 7:e21. https:// doi.org/10.1016/S2215-0366(20)30090-0

3. Colodro-Conde L, Couvy-Duchesne B, Zhu G et al (2018) A direct test of the diathesis-stress model for depression. Mol Psychiatry 23:1590-1596. https://doi.org/10.1038/mp.2017.130

4. Robinson E, Sutin AR, Daly M, Jones A (2021) A systematic review and meta-analysis of longitudinal cohort studies comparing mental health before versus during the COVID-19 pandemic. medRxiv. https://doi.org/10.1101/2021.03.04.21252921

5. Armitage R, Nellums LB (2020) COVID-19 and the consequences of isolating the elderly. Lancet Public Health 5:e235. https://doi. org/10.1016/S2468-2667(20)30061-X

6. Santini ZI, Jose PE, York Cornwell E et al (2020) Social disconnectedness, perceived isolation, and symptoms of depression and anxiety among older Americans (NSHAP): a longitudinal 
mediation analysis. Lancet Public Health 5:E62-E70. https://doi. org/10.1016/S2468-2667(19)30230-0

7. Czeisler MÉ, Lane RI, Petrosky E et al (2020) Mental health, substance use, and suicidal ideation during the COVID-19 pandemic - United States, June 24-30, 2020. Morb Mortal Wkly Rep 69:1049-1057

8. González-Sanguino C, Ausín B et al (2020) Mental health consequences during the initial stage of the 2020 Coronavirus pandemic (COVID-19) in Spain. Brain Behav Immun 87:172-176. https:// doi.org/10.1016/j.bbi.2020.05.040

9. Röhr S, Reininghaus U, Riedel-Heller SG (2020) Mental wellbeing in the German old age population largely unaltered during COVID-19 lockdown: results of a representative survey. BMC Geriatr 20:489. https://doi.org/10.1186/s12877-020-01889-x

10. Wang H, Li T, Barbarino P et al (2020) Dementia care during COVID-19. Lancet 395:1190-1191. https://doi.org/10.1016/ S0140-6736(20)30755-8

11. Manca R, De Marco M, Venneri A (2020) The impact of COVID19 infection and enforced prolonged social isolation on neuropsychiatric symptoms in older adults with and without dementia: a review. Front Psychiatry 11:585540. https://doi.org/10.3389/fpsyt. 2020.585540

12. Mok VCT, Pendlebury S, Wong A et al (2020) Tackling challenges in care of Alzheimer's disease and other dementias amid the COVID-19 pandemic, now and in the future. Alzheimers Dement 16:1571-1581. https://doi.org/10.1002/alz.12143

13. Hamm ME, Brown PJ, Karp JF et al (2020) Experiences of American older adults with pre-existing depression during the beginnings of the COVID-19 pandemic: a multicity, mixed-methods study. Am J Geriatr Psychiatry 28:924-932. https://doi.org/10. 1016/j.jagp.2020.06.013

14. Li W, Zhao N, Yan X et al (2021) The prevalence of depressive and anxiety symptoms and their associations with quality of life among clinically stable older patients with psychiatric disorders during the COVID-19 pandemic. Transl Psychiatry 11:75. https:// doi.org/10.1038/s41398-021-01196-y

15. Topp CW, Østergaard SD, Søndergaard S, Bech P (2015) The WHO-5 well-being index: a systematic review of the literature. Psychother Psychosom 84:167-176. https://doi.org/10.1159/ 000376585

16. Conrad I, Matschinger H, Riedel-Heller S, von Gottberg C, Kilian $R$ (2014) The psychometric properties of the German version of the WHOQOL-OLD in the German population aged 60 and older. Health Qual Life Outcomes 12:105

17. Conrad I, Matschinger H, Kilian R, Riedel-Heller SG (2016) WHOQOL-OLD und WHOQOL-BREF: Manual: Handbook for the German-language versions of the WHO instruments for assessing quality of life in old age (German). Hogrefe, Göttingen

18. Spitzer RL, Kroenke K, Williams JB, Löwe B (2006) A brief measure for assessing generalized anxiety disorder: the GAD-7. Arch Intern Med 166:1092-1097
19. Ahrendt D, Anderson R, Dubois H et al (2017) European Quality of Life Survey 2016: Quality of life, quality of public services, and quality of society. Publications Office of the European Union, Eurofound, Luxembourg

20. Hinz A, Klein AM, Brähler E et al (2017) Psychometric evaluation of the Generalized Anxiety Disorder Screener GAD-7, based on a large German general population sample. J Affect Disord 210:338-344. https://doi.org/10.1016/j.jad.2016.12.012

21. Hussenoeder FS, Jentzsch D, Matschinger H et al (2021) Depression and quality of life in old age: a closer look. Eur J Ageing 18:75-83. https://doi.org/10.1007/s10433-020-00573-8

22. Vahia IV, Jeste DV, Reynolds CF (2020) Older adults and the mental health effects of COVID-19. JAMA 32:2253-2254

23. Kuehner C, Schultz K, Gass P, Meyer-Lindenberg A, Dreßing H (2020) Mental health status in the community during the COVID19-pandemic (German). Psychiatr Prax 47:361-369

24. Bäuerle A, Teufel M, Musche V et al (2020) Increased generalized anxiety, depression and distress during the COVID-19 pandemic: a cross-sectional study in Germany. J Public Health 42:1-7. https://doi.org/10.1093/pubmed/fdaa106

25. García-Fernández L, Romero-Ferreiro V, López-Roldán PD, Padilla S, Rodriguez-Jimenez R (2020) Mental health in elderly Spanish people in times of COVID-19 outbreak. Am J Geriatr Psychiatry 28:1040-1045. https://doi.org/10.1016/j.jagp.2020.06. 027

26. van Tilburg TG, Steinmetz S, Stolte E, van der Roest H, de Vries DH (2020) Loneliness and mental health during the COVID-19 pandemic: a study among Dutch older adults. J Gerontol B Psychol Sci Soc Sci 76:e249-e255. https://doi.org/10.1093/geronb/ gbaa111

27. Bidzan-Bluma I, Bidzan M, Jurek P et al (2020) A Polish and German population study of quality of life, well-being, and life satisfaction in older adults during the COVID-19 pandemic. Front Psychiatry 11:585813. https://doi.org/10.3389/fpsyt.2020.585813

28. Banerjee S, Samsi K, Petrie CD et al (2009) What do we know about quality of life in dementia? A review of the emerging evidence on the predictive and explanatory value of disease specific measures of health related quality of life in people with dementia. Int J Geriatr Psychiatry 24:15-24. https://doi.org/10.1002/gps. 2090

29. Farina N, King D, Burgon C et al (2020) MODEM group. Disease severity accounts for minimal variance of quality of life in people with dementia and their carers: analyses of cross-sectional data from the MODEM study. BMC Geriatr 20:232. https://doi.org/10. 1186/s12877-020-01629-1

30. Martyr A, Nelis SM, Quinn C et al (2018) Living well with dementia: a systematic review and correlational meta-analysis of factors associated with quality of life, well-being and life satisfaction in people with dementia. Psychol Med 48:2130-2139. https:// doi.org/10.1017/S0033291718000405 